\title{
ZEROS AND POLES OF FUNCTIONS DEFINED BY TAYLOR SERIES
}

\author{
MICHAEL GOLOMB
}

1. Introduction. With the objective of providing a straightforward numerical method for the determination of poles and zeros of functions defined by Taylor series this note reexamines Hadamard's solution of this problem, which is found in his classical thesis. ${ }^{1}$ The best known part of Hadamard's solution is the criterion which enables one to determine the meromorphic character of the expanded function and the total number of poles on the circle of convergence. But this solution also includes a method of determining these poles as functions of the Taylor coefficients, and Hadamard himself intimated that his results should prove useful in the numerical evaluation of poles and zeros. However, it seems that, as a device in numerical analysis, his method has attracted much less attention than it deserves. This may be due to the fact that Hadamard's criterion for the number of poles employs limits superior, which are impractical for numerical work.

In this paper no use is made of limits superior, and the number of poles on the circle of convergence is ascertained by the process of evaluating their affixes. Besides determining the polynomial whose zeros are all the poles of the expanded function on the circle of convergence with their proper multiplicities, the paper also determines the polynomial whose zeros are the different poles of highest order only. These results are based on an identity and an inequality for persymmetric determinants involving successive Taylor coefficients of rational and meromorphic functions, which seem to be new, and may also prove useful in other applications.

2. A formula for persymmetric determinants. We first are going to establish an identity for persymmetric determinants of the form

$$
d_{n}^{(m)}=\left|\begin{array}{cccc}
c_{n+1} & c_{n+2} & \cdots & c_{n+m} \\
c_{n+2} & c_{n+3} & \cdots & c_{n+m+1} \\
. & \cdot & \cdots & . \\
c_{n+m} & c_{n+m+1} & \cdots & c_{n+2 m-1}
\end{array}\right|, \quad n=-1,0,1, \cdots
$$

Received by the editors August 7, 1942.

$1 \mathrm{~J}$. Hadamard, Essai sur l'êtude des fonctions données par leur développement de Taylor, J. Math. Pures Appl. (4) vol. 8 (1892) pp. 101-186. 
where the $2 m-1$ numbers $c_{n+1}, c_{n+2}, \cdots, c_{n+2 m-1}$ are successive coefficients in the Taylor series expansion of a rational function.

Suppose, at first, the rational function has only simple poles and is of the form $g_{0}(z)=\sum_{n=0}^{\infty} c_{n} z^{n}=-\sum_{k=1}^{p} \zeta_{k} /\left(z-z_{k}\right), z_{k} \neq 0$. We wish to evaluate $d_{n}^{(p)}=d_{n}^{(p)}\left[g_{0}\right]$. Since $c_{n}=\sum_{k=1}^{p} \zeta_{k} z_{k}^{-(n+1)}(n=0,1, \cdots)$, we have

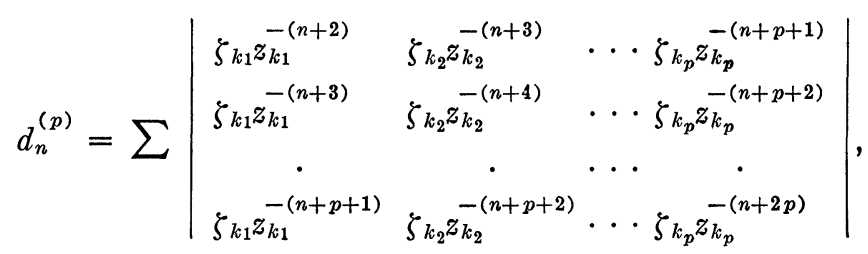

where the sum is extended over all the combinations (with repetitions) $k_{1}, k_{2}, \cdots, k_{p}$ of the numbers $1,2, \cdots, p$. Since the combinations with repeated elements lead to vanishing determinants we may also write

$$
d_{n}^{(p)}=\frac{\zeta_{1} \zeta_{2} \cdots \zeta_{p}}{\left(z_{1} z_{2} \cdots z_{p}\right)^{n+2 p}} \sum\left|\begin{array}{cccc}
z_{k_{1}}^{2 p-2} & z_{k_{2}}^{2 p-3} & \cdots & z_{k_{p}}^{p-1} \\
z_{k_{1}}^{2 p-3} & z_{k_{2}}^{2 p-4} & \cdots & z_{k_{p}}^{p-2} \\
\cdot & \cdot & \cdots & \cdot \\
z_{k_{1}}^{p-1} & z_{k_{2}}^{p-2} & \cdots & z_{k_{p}}^{0}
\end{array}\right|
$$

The sum of determinants in the last formula is known to be equal to the square of the difference-product of the numbers $z_{1}, z_{2}, \cdots, z_{p}$; hence

$$
\begin{aligned}
d_{n}^{(p)}\left[g_{0}\right] & =\Delta \zeta_{1} \zeta_{2} \cdots \zeta_{p}\left(z_{1} z_{2} \cdots z_{p}\right)^{-(n+2 p)}, \\
\Delta & =\prod_{k<k^{\prime}}\left(z_{k}-z_{k^{\prime}}\right)^{2}, \quad k, k^{\prime}=1,2, \cdots, p .
\end{aligned}
$$

Now suppose the $2 p-1$ numbers $c_{n+1}, c_{n+2}, \cdots, c_{n+2 p-1}$ are successive Taylor coefficients of the more general rational function

$$
\begin{aligned}
& g(z)=\sum_{n=0}^{\infty} c_{n} z^{n} \\
&=-\sum_{k=1}^{q}\left(\frac{\zeta_{k}}{\left(z-z_{k}\right)^{p_{k}}}+\frac{\zeta_{k, 1}}{\left(z-z_{k}\right)^{p_{k}-1}}+\cdots+\frac{\zeta_{k, p_{k}-1}}{z-z_{k}}\right), \\
& z_{k} \neq 0,
\end{aligned}
$$

which has, at most, $p=p_{1}+p_{2}+\cdots+p_{q}$ poles (counted according to their multiplicities). We are going to evaluate $d_{n}^{(p)}=d_{n}^{(p)}[g]$ as the 
limit of determinants $d_{n}^{(p)}$ formed for rational functions with only simple poles.

With the numbers $z_{k, 1}, z_{k, 2}, \cdots, z_{k, p_{k}}(k=1,2, \cdots, q)$ which are assumed to be different from each other and from 0 but otherwise arbitrary, we define the polynomial

$$
\pi_{k}(z)=\zeta_{k}+\zeta_{k, 1}\left(z-z_{k}\right)+\cdots+\zeta_{k, p_{k-1}}\left(z-z_{k}\right)^{p_{k-1}},
$$

and consider, instead of the function $g(z)$, the function

$$
g_{1}(z)=\sum_{n=0}^{\infty} c_{n}^{\prime} z^{n}=-\sum_{k=1}^{q} \frac{\pi_{k}(z)}{\left(z-z_{k, 1}\right)\left(z-z_{k, 2}\right) \cdots\left(z-z_{k, p_{k}}\right)} \cdot
$$

$g_{1}(z)$ has only simple poles. Its partial fraction expansion is

$$
g_{1}(z)=-\sum_{k, l} \frac{\zeta_{k, l}^{\prime}}{z-z_{k, l}}, \quad l=1,2, \cdots, p_{k} ; k=1,2, \cdots, q,
$$

where

$$
\zeta_{k, l}^{\prime}=\frac{\pi_{k}\left(z_{k, l}\right)}{\left(z_{k, l}-z_{k, 1}\right) \cdots\left(z_{k, l}-z_{k, l-1}\right)\left(z_{k, l}-z_{k, l+1}\right) \cdots\left(z_{k, l}-z_{k, p_{k}}\right)} .
$$

Applying the first result (see formula (2)) to the function $g_{1}(z)$, we find $d_{n}^{(p)}\left[g_{1}\right]=\Delta^{\prime} \prod_{k, l} \zeta_{k, l}^{\prime} z_{k, l}^{-(n+2 p)}$, where $\Delta^{\prime}$ is the square of the difference-product of the numbers $z_{k, l}$. But $\prod_{k, l} \xi_{k, l}^{\prime}=\prod_{k, l} \pi_{k}\left(z_{k, l}\right) / \prod_{k} \Delta_{k}$, where $\Delta_{k}=(-1)^{p_{k}\left(p_{k}-1\right) / 2} \prod_{l<l^{\prime}}\left(z_{k, l}-z_{k, l^{\prime}}\right)^{2}$. Hence

$$
\begin{aligned}
d_{n}^{(p)}\left[g_{1}\right] & =\Delta^{\prime \prime} \prod_{k, l} \pi_{k}\left(z_{k, l}\right) z_{k, l}^{-(n+2 p)}, \\
\Delta^{\prime \prime} & =(-1)^{\Sigma_{k} p_{k}\left(p_{k}-1\right) / 2} \prod_{k<l^{\prime} ; l, l^{\prime}}\left(z_{k, l}-z_{k^{\prime}, l^{\prime}}\right)^{2}, \\
l & =1,2, \cdots, p_{k} ; l^{\prime}=1,2, \cdots, p_{k^{\prime}} .
\end{aligned}
$$

We now assume that the numbers $z_{k, l}$ depend on a variable $\delta$, and that $\lim _{\delta \rightarrow 0} z_{k, l}(\delta)=z_{k}\left(l=1,2, \cdots, p_{k} ; k=1,2, \cdots, q\right)$. Instead of $g_{1}(z)$ and $c_{n}^{\prime}$ we now have $g(z, \delta)$ and $c_{n}(\delta)$ respectively. Comparing (5) and (3), we see that $g(z, \delta)$ converges to $g(z), c_{n}(\delta)$ converges to $c_{n}$, as $\delta \rightarrow 0$. Hence $d_{n}^{(p)}[g(\delta)]$ converges to $d_{n}^{(p)}[g]$, as $\delta \rightarrow 0$. Moreover, by (4), $\pi_{k}\left(z_{k, l}\right)$ converges to $\zeta_{k}$, as $\delta \rightarrow 0$. Using all these limits, we obtain from (6)

$$
\begin{aligned}
d_{n}^{(p)}[g] & =\Delta \zeta_{1}^{p_{1}} \zeta_{2}^{p_{2}} \cdots \zeta_{q}^{p_{q}}\left(z_{1}^{p_{1}} z_{2}^{p_{2}} \cdots z_{q}^{p_{q}}\right)^{-(n+2 p)}, \\
\Delta & =(-1)^{\Sigma_{k} p_{k}\left(p_{k}-1\right) / 2} \prod_{k<k^{\prime}}\left(z_{k}-z_{k^{\prime}}\right)^{2 p_{k} p_{k^{\prime}}}, k, k^{\prime}=1,2, \cdots, q .
\end{aligned}
$$


3. The equation for all the poles on the circle of convergence. In the following, the numbers $a_{n}$ will represent the Taylor coefficients of any analytic function that has no singularities other than poles on its circle of convergence about 0 . And besides such determinants as defined in (1), we shall consider determinants $d_{n i}^{(m)}(i=1,2, \cdots, m)$, defined as

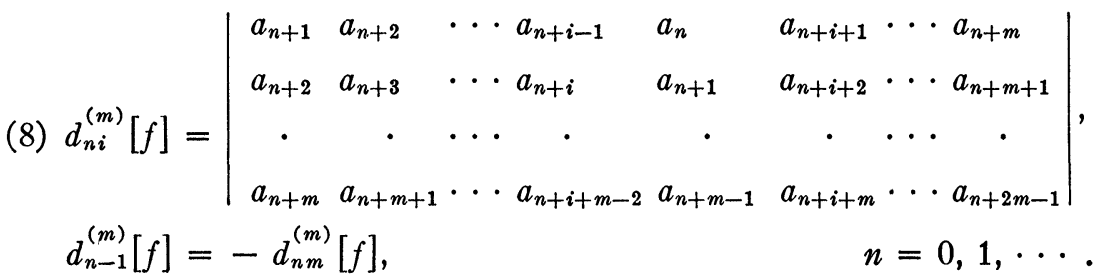

ThEOREM 1. Suppose $f(z)=\sum_{n} a_{n} z^{n}=g(z)+f_{1}(z)$, where

$$
\begin{aligned}
& g(z)=-\sum_{k=1}^{q}\left(\frac{\zeta_{k}}{\left(z-z_{k}\right)^{p_{k}}}+\frac{\zeta_{k, 1}}{\left(z-z_{k}\right)^{p_{k-1}}}+\cdots+\frac{\zeta_{k, p_{k}-1}}{z-z_{k}}\right), \\
& \left|z_{k}\right|=r>0, \quad z_{k} \neq z_{k^{\prime}}\left(k \neq k^{\prime}\right), \quad \zeta_{k} \neq 0, \quad k, k^{\prime}=1,2, \cdots, q,
\end{aligned}
$$

and $f_{1}(z)$ is regular in $|z| \leqq R=\tau r, \tau>1$; suppose

$$
\begin{aligned}
\pi(z) & =\left(z-z_{1}\right)^{p_{1}}\left(z-z_{2}\right)^{p_{2}} \cdots\left(z-z_{q}\right)^{p_{q}} \\
& =z^{p}+\gamma_{1} z^{p-1}+\cdots+\gamma_{p}, \quad p=p_{1}+p_{2}+\cdots+p_{q} .
\end{aligned}
$$

Then, for $i=1,2, \cdots, p$,

$$
\gamma_{i}+d_{n i}^{(p)}[f] / d_{n}^{(p)}[f]=O\left(\tau^{-n}\right), \quad \text { as } n \rightarrow \infty .
$$

Proof. The function $\phi(z)=\sum_{\nu} \alpha_{\nu} z^{\nu}=\pi(z) f(z)$ has the coefficients

$$
\alpha_{\nu}=a_{\nu-p}+\gamma_{1} a_{\nu-p+1}+\gamma_{2} a_{\nu-p+2}+\cdots+\gamma_{p} a_{\nu} .
$$

If, in (10), we put $\nu$ successively equal to $n+p, n+p+1, \cdots$, $n+2 p-1$, we have $p$ equations for the unknowns $\gamma_{1}, \gamma_{2}, \cdots, \gamma_{p}$, whose solution is, provided $d_{n}^{(p)}[f] \neq 0$,

$$
\gamma_{i}+d_{n i}^{(p)} / d_{n}^{(p)}=e_{n i}^{(p)} / d_{n}^{(p)}, \quad i=1,2, \cdots, p,
$$

where $e_{n i}^{(p)}$ denotes the determinant obtained from $d_{n i}^{(p)}$ when $a_{n}$, $a_{n+1}, \cdots, a_{n+p-1}$ are replaced by $\alpha_{n+p}, \alpha_{n+p+1}, \cdots, \alpha_{n+2 p-1}$ respectively.

The determinants $e_{n i}^{(p)}$ are easily estimated. Since $\phi(z)$ is regular in $|z| \leqq R$, and $f(z)$ is regular in $|z|<r$, there is some number $\sigma>1$ 
such that $\alpha_{n}=O\left(\sigma^{-n} R^{-n}\right), a_{n}=O\left(\sigma^{n /(p-1)} r^{-n}\right)$ as $n \rightarrow \infty$; hence

$$
e_{n i}^{(p)}=O\left(r^{-(p-1) n} R^{-n}\right)=O\left(\tau^{-n} r^{-p n}\right), \quad \text { as } n \rightarrow \infty .
$$

A lower bound for the determinants $d_{n}^{(p)}[f]$ is derived from identity (7). If $g(z)=\sum_{n} c_{n} z^{n}, f_{1}(z)=\sum_{n} b_{n} z^{n}$, then $a_{n}=b_{n}+c_{n}$, and $d_{n}^{(p)}[f]$ is the sum of $d_{n}^{(p)}[g]$ and several other determinants, each of which has at least one column of $b$ 's and otherwise columns of $c$ 's. Hence, as in (12),

$$
d_{n}^{(p)}[f]=d_{n}^{(p)}[g]+O\left(\tau^{-n} r^{-p n}\right), \quad \text { as } n \rightarrow \infty .
$$

But since, by (7), for $n=0,1,2, \cdots,\left|d_{n}^{(p)}[g]\right|=M r^{-p n}$, where $M$ is a positive constant, we deduce from (13) that there is another constant $M_{1}$ such that, for sufficiently large $n$,

$$
\left|d_{n}^{(p)}[f]\right|>M_{1} r^{-p n}
$$

In particular, $d_{n}^{(p)}[f] \neq 0$ when $n$ is sufficiently large. •

Inequalities (12) and (14), together with equation (11), prove the theorem.

Let it be noted that, since $\gamma_{p}=(-1)^{p} z_{1}^{p_{1}} z_{2}^{p_{2}} \cdots z_{q}^{p_{q}}$, we have $r^{p}=\left|\gamma_{p}\right|$, or by $(9), r^{p}=\lim _{n \rightarrow \infty}\left|d_{n-1}^{(p)}[f] / d_{n}^{(p)}[f]\right|$. This relation implies $r^{p}=\lim _{n \rightarrow \infty}\left|d_{n}^{(p)}[f]\right|-1 / n .^{2}$

It is not necessary that all the poles $z_{k}$ should lie on the circle $|z|=r$. Indeed, we may state the following:

Corollary. Estimate (9) still holds true if $\left|z_{k}\right|=\tau_{k} r, \tau_{k} \geqq 1$ $(k=1,2, \cdots, q)$, and $R=\tau r \cdot \tau_{1}^{p_{1}} \tau_{2}^{p_{2}} \cdots \tau_{q}^{p_{q}}, \tau>1$.

The proof of this corollary is an obvious variation of the proof given above.

If $f(z)$ is a rational function, say $f(z)=\psi(z) / \pi(z)$, where $\psi(z)$ is a polynomial whose degree we define to be $s \geqq p-2$, then $\phi(z)=\sum_{\nu} \alpha_{\nu} z^{\nu}$ $=\pi(z) f(z)=\psi(z)$; hence $\alpha_{\nu}=0$ for $\nu>s$, and consequently $e_{n i}^{(p)}=0$ for $n>s-p$. On the other hand, $f(z)$ is the sum of a polynomial $f_{1}(z)$ of degree $s-p$ and a fraction $g(z)$ such as in (3). Hence, for $n>s-p$, $d_{n}^{(p)}[f]=d_{n}^{(p)}[g] \neq 0$, by (7). Thus, relation (9) holds, for $n>s-p$, with 0 substituted for the second term. In particular,

2 This "rather delicate limit relation" (see P. Dienes, The Taylor series, Oxford,1931, p. 330) was first proved by Hadamard, op. cit. Other authors have tried to give simpler proofs for it, see, for example, A. Ostrowski, Über einen Satz von Herrn Hadamard, Jber. Deutscher Math. Verein. vol. 35 (1926) pp. 179-182. It is an immediate consequence of identity (7). On the other hand, it is the only intricate point in the proof of Theorem 1. 


$$
\gamma_{i}=-d_{s-p+1, i}^{(p)}[f] / d_{s-p+1}^{(p)}[f], \quad i=1,2, \cdots, p .
$$

The numbers $\gamma_{i}$ having been found, the numbers $\alpha_{\nu}$ are given by (10). Thus, the coefficients of the polynomials $\pi(z)$ and $\psi(z)$ are expressed, by (15) and (10), as rational functions of the Taylor coefficients $a_{0}, a_{1}, \cdots, a_{p+s}$ of $f(z)=\psi(z) / \pi(z)$.

4. An inequality for persymmetric determinants. In the following as in the foregoing we shall assume the function $f(z)=\sum_{n} a_{n} z^{n}$ to have no singularities other than poles on the circle of convergence $|z|=r$ of the Taylor series. We shall be interested no longer in all the poles, but only in the poles of highest order on $|z|=r$. We shall say that $f(z)$ is of order $m$ on $|z|=r$ if $f(z)$ has there at least one pole of order $m$, and no other singularities but poles of order less than or equal to $m$.

We are going to establish an estimate for the determinants $d_{n}^{(q)}[f]$ $(n=0,1, \ldots)$ where $q$ is the number of poles of highest order of $f(z)$ on $|z|=r$. Suppose that $f(z)=\sum_{n} a_{n} z^{n}=g(z)+f_{1}(z)$, where $g(z)$ $=-\sum_{k=1}^{q} \xi_{k} /\left(z-z_{k}\right)^{m},\left|z_{k}\right|=r>0$, and $f_{1}(z)$ is regular in $|z|<r$ and of order less than $m$ on $|z|=r$. The coefficient of $z^{n}$ in the Taylor series expansion of $-\zeta_{k}\left(z-z_{k}\right)^{-m}$ is

$$
\begin{aligned}
& (-1)^{m-1} \frac{(n+1)(n+2) \cdots(n+m-1)}{(m-1) ! z_{k}^{m+n}} \zeta_{k} \\
& =(-1)^{m-1} \frac{n^{m-1}}{(m-1) ! z_{k}^{m+n}} \zeta_{k}+O\left(\frac{n^{m-2}}{r^{n}}\right), \quad \text { as } n \rightarrow \infty .
\end{aligned}
$$

The coefficient of $z^{n}$ in the Taylor series expansion of $f_{1}(z)$ is, for the same reason, $O\left(n^{m-2} r^{-n}\right)$. Hence we have the estimate

$$
\begin{aligned}
a_{n} & =\sum_{k=1}^{q} \frac{\xi_{k}}{z_{k}^{n+1}}+O\left(\frac{n^{m-2}}{r^{n}}\right), \\
\xi_{k} & =(-1)^{m-1} \frac{n^{m-1}}{(m-1) ! z_{k}^{m-1}} \zeta_{k} .
\end{aligned}
$$

as $n \rightarrow \infty$,

It implies the less exact estimate

$$
a_{n}=O\left(n^{m-1} / r^{n}\right), \quad \text { as } n \rightarrow \infty .
$$

Corresponding to the decomposition (16), the determinant $d_{n}^{(q)}[f]$ splits into the sum of a persymmetric determinant whose terms are $c_{\nu}=\sum_{k=1}^{q} \xi_{k} z_{k}^{-(\nu+1)}(\nu=n+1, n+2, \cdots, n+2 q-1)$ and several other determinants, each of which has at least one column made of terms that are $O\left(n^{m-2} r^{-n}\right)$, and otherwise columns made of terms that are 
$O\left(n^{m-1} \gamma^{-n}\right)$. The first determinant, as was shown in $\$ 2$, is equal to $\Delta \cdot \xi_{1} \xi_{2} \cdots \xi_{q}\left(z_{1} z_{2} \cdots z_{q}\right)^{-(n+2 q)}$, where $\Delta$ is the square of the difference-product of the numbers $z_{1}, z_{2}, \cdots, z_{q}$. The sum of the other determinants is $O\left(n^{q(m-1)-1} r^{q n}\right)$. Hence, using (16), we have the estimate

$$
\begin{aligned}
d_{n}^{(q)}[f] & =C_{f} \frac{n^{q(m-1)}}{\left(z_{1} z_{2} \cdots z_{q}\right)^{n}}+O\left(\frac{n^{q(m-1)-1}}{r^{q n}}\right), \text { as } n \rightarrow \infty, \\
C_{f} & =(-1)^{q(m-1)} \Delta \cdot \frac{\zeta_{1} \zeta_{2} \cdots \zeta_{q}}{[(m-1) !]^{q}\left(z_{1} z_{2} \cdots z_{q}\right)^{2 q+m-1}} .
\end{aligned}
$$

5. The equation for the poles of highest order. In $\$ 3$ we have established the algebraic equation whose roots are all the poles of $f(z)$ (counted according to their multiplicities) on $|z|=r$. We shall now establish the algebraic equation whose roots are the different poles of highest order of $f(z)$ on $|z|=r$.

THEOREM 2. Suppose that $f(z)=\sum_{n} a_{n} z^{n}=g(z)+f_{1}(z)$, where

$$
\begin{aligned}
g(z) & =-\left(\zeta_{1} /\left(z-z_{1}\right)^{m}+\zeta_{2} /\left(z-z_{2}\right)^{m}+\cdots+\zeta_{q} /\left(z-z_{q}\right)^{m}\right), \\
\left|z_{k}\right| & =r>0, \quad z_{k} \neq z_{k^{\prime}}\left(k \neq k^{\prime}\right), \quad \zeta_{k} \neq 0, \quad k, k^{\prime}=1,2, \cdots, q,
\end{aligned}
$$

and $f_{1}(z)$ is regular in $|z|<r$ and of order less than $m$ on $|z|=r$; suppose $\pi(z)=\left(z-z_{1}\right)\left(z-z_{2}\right) \cdots\left(z-z_{q}\right)=z^{q}+\gamma_{1} z^{q-1}+\cdots+\gamma_{q}$. Then, for $i=1,2, \cdots, q$,

$$
\gamma_{i}+d_{n i}^{(q)}[f] / d_{n}^{(q)}[f]=O(1 / n), \quad \text { as } n \rightarrow \infty,
$$

and

$$
m=1+\lim _{n \rightarrow \infty} \frac{\log \left|d_{n}^{(q)}[f]\right|+n q \log r}{q \log n}
$$

PROOF. The function $\phi(z)=\sum_{\nu} \alpha_{\nu} z^{\nu}=\pi(z) f(z)$, whose coefficients are

$$
\alpha_{\nu}=a_{\nu-q}+\gamma_{1} a_{\nu-q+1}+\cdots+\gamma_{q} a_{\nu},
$$

is regular in $|z|<r$, and of order less than or equal to $m-1$ on $|z|=r$. Hence, as in (17), the coefficients $\alpha_{\nu}$ are $O\left(\nu^{m-2} r^{-\nu}\right)$, as $\nu \rightarrow \infty$. If, in

${ }^{3}$ Hadamard, see op. cit., defined the order of any series $\sum a_{n} z^{n}$ and proved that, on its circle of convergence, the order is given by the formula $m=1+\lim \sup \left(\log \left|a_{n}\right|\right.$ $+n \log r) / \log n$. Formula (19b) represents, in accordance with the objective of this paper, the order as a regular limit, but it holds only for series which are meromorphic on their circle of convergence. 
(20), we put $\nu$ successively equal to $n+q, n+q+1, \cdots, n+2 q-1$, we have $q$ equations for the unknowns $\gamma_{1}, \gamma_{2}, \cdots, \gamma_{g}$, whose solution is, provided $d_{n}^{(q)}[f] \neq 0$,

$$
\gamma_{i}+d_{n i}^{(q)} / d_{n}^{(q)}=e_{n i}^{(q)} / d_{n}^{(q)}, \quad i=1,2, \cdots, q,
$$

where $e_{n i}^{(q)}$ denotes the determinant that is obtained from $d_{n i}^{(q)}$ when $a_{n}, a_{n+1}, \cdots, a_{n+q-1}$ are replaced by $\alpha_{n+q}, \alpha_{n+q+1}, \cdots, \alpha_{n+2 q-1}$ respectively. Using estimate (17) for the $a$ 's and a corresponding estimate for the $\alpha$ 's, we find readily

$$
e_{n i}^{(q)}=O\left(n^{q(m-1)-1} r^{-q n}\right), \quad \quad \text { as } n \rightarrow \infty .
$$

Taking into account that, by assumption, the poles $z_{1}, z_{2}, \cdots, z_{q}$ are different from each other, we deduce from relation (18) that there are constants $M_{1}, M_{2}$ such that, for sufficiently large $n$,

$$
d_{n}^{(q)}[f]=\mu_{n} n^{q(m-1)} r^{-q n}, \quad M_{1}<\left|\mu_{n}\right|<M_{2} .
$$

In particular, $d_{n}^{(q)}[f] \neq 0$ when $n$ is sufficiently large.

Inequalities (22) and (23), together with equation (21), prove the theorem.

6. Converses of Theorems 1 and 2. In problems where the poles of $f(z)$ are to be determined from the given Taylor coefficients, it is, in general, not known, a priori, what and how many singularities $f(z)$ has on the circle of convergence $|z|=r$ of its Taylor series expansion. Even if $f(z)$ is known to be meromorphic, Theorems 1 and 2 do not always suffice to establish the polynomial $\pi(z)$ whose zeros are all the poles, or the poles of highest order, of $f(z)$ on $|z|=r$, as the numbers $p$ or $q$ may not be known. In such a case the procedure to follow is to investigate the behavior of the quotients $d_{n i}^{m}[f] / d_{n}^{(m)}[f]$, as $n \rightarrow \infty$, successively for $m=1,2, \cdots$. From the convergence properties of these sequences the nature and number of the singularities of $f(z)$ on $|z|=r$ can be deduced, as will be shown in the following converses of Theorems 1 and 2. It will always be assumed that $f(z)=\sum_{n} a_{n} z^{n}$ has a positive radius of convergence.

Theorem 3. Suppose that, for a fixed $p$, there are $p$ numbers $\gamma_{1}, \gamma_{2}, \cdots, \gamma_{p}$ and a number $\tau>1$ such that, for $i=1,2, \cdots, p$,

$$
\gamma_{i}+d_{n i}^{(p)}[f] / d_{n}^{(p)}[f]=O\left(\tau^{-n}\right), \quad \text { as } n \rightarrow \infty ;
$$

suppose that this is not the case for any $p^{\prime}<p$. Then $f(z)$ is regular in 
the circle $|z| \leqq r=\left|\gamma_{p}\right|^{1 / p}$ but for $p$ poles (counted according to their multiplicities) on $|z|=r$, which are the zeros of the polynomials $\pi(z)$ $=z^{p}+\sum_{i=1}^{p} \gamma_{i} z^{p-i}$.

Proof. According to the definition of the determinants $d_{n i}^{(p)}, d_{n}^{(p)}$, and since the quotients $d_{n i}^{(p)} / d_{n}^{(p)}$ exist, by assumption, for sufficiently large $n$, we have

$$
a_{n}-\left(1 / d_{n}^{(p)}\right)\left(a_{n+1} d_{n 1}^{(p)}+a_{n+2} d_{n 2}^{(p)}+\cdots+a_{n+p} d_{n p}^{(p)}\right)=0 .
$$

Let $r$ be the radius of convergence of $\sum_{n} a_{n} z^{n}$; then $a_{n}=O\left(r^{\prime-n}\right)$, as $n \rightarrow \infty$, for any $r^{\prime}<r$. If we choose $r^{\prime}$ so that $R=\tau r^{\prime}>r$, we obtain, by substituting (24) in (25),

$$
\alpha_{n+p}=a_{n}+\gamma_{1} a_{n+1}+\gamma_{2} a_{n+2}+\cdots+\gamma_{p} a_{n+p}=O\left(R^{-n}\right) \text {, as } n \rightarrow \infty \text {. }
$$

Hence the function $\phi(z)=\pi(z) f(z)=\sum_{\nu} \alpha_{\nu} z^{\nu}$ is regular in $|z| \leqq R>r$. This implies that $f(z)$ has no other singularities than, at most, $p$ poles on the circle $|z|=r$, which are among the zeros of $\pi(z)$.

If $f(z)$ had less than $p$ poles on $|z|=r$, some relation like (24) would, by Theorem 1 , hold for $p^{\prime}<p$, in contradiction with the hypothesis. Hence $f(z)$ has exactly $p$ poles on $|z|=r$, which are the zeros of $\pi(z)$. Since $(-1)^{p} \gamma_{p}$ is the product of the $p$ zeros, $r=\left|\gamma_{p}\right|^{1 / p}$.

THEOREM 4. Suppose $f(z)$ has no singularities but poles on $|z|=r$; suppose that, for a fixed $q$, there are $q$ numbers $\gamma_{1}, \gamma_{2}, \cdots, \gamma_{q}$ such that, for $i=1,2, \cdots, q$,

$$
\gamma_{i}+\lim _{n \rightarrow \infty} d_{n i}^{(q)}[f] / d_{n}^{(q)}[f]=0 ;
$$

suppose that this is not the case for any $q^{\prime}<q$. Then $f(z)$ is regular in the circle $|z|<r=\left|\gamma_{q}\right|^{1 / q}$, and of order

$$
m=1+\lim _{n \rightarrow \infty}\left(\log \left|d_{n}^{(q)}\right|+n q \log r\right) / q \log n
$$

on $|z|=r . f(z)$ has $q$ different poles of order $m$ on $|z|=r$, which are the zeros of the polynomial $\pi(z)=z^{q}+\sum_{i=1}^{q} \gamma_{i} z^{q-i}$.

Proof. Let $r$ be the radius of convergence of $\sum_{n} a_{n} z^{n}$, and let $m$ be the order of $f(z)$ on $|z|=r$. If $f(z)$ had less than $q$ poles on $|z|=r$, some relation like (26) would, by Theorem 2 , hold true for $q^{\prime}<q$, in contradiction with the hypothesis. Hence $f(z)$ has $q^{\prime} \geqq q$ poles of order $m$ on $|z|=r$.

As in the proof of Theorem 3, we have, for sufficiently large $n$,

$$
a_{n}-\left(1 / d_{n}^{(q)}\right)\left(a_{n+1} d_{n 1}^{(q)}+a_{n+2} d_{n 2}^{(q)}+\cdots+a_{n+q} d_{n q}^{(q)}\right)=0 .
$$


In $\S 4$ (see (17)) it was shown that $a_{n}=O\left(n^{m-1} \gamma^{-n}\right)$, as $n \rightarrow \infty$, whereas by assumption, $d_{n i}^{(a)} / d_{n}^{(a)}=-\gamma_{i}+o(1)$, as $n \rightarrow \infty$. Hence (27) implies

$$
\alpha_{n+q}=a_{n}+\gamma_{1} a_{n+1}+\gamma_{2} a_{n+2}+\cdots+\gamma_{q} a_{n+q}=o\left(n^{m-1} r^{-n}\right),
$$

as $n \rightarrow \infty$.

Now suppose $q^{\prime}>q$. Since $\pi(z)$ is of degree $q, \phi(z)=\sum_{n} \alpha_{n} z^{n}$ $=\pi(z) f(z)$ is, like $f(z)$, of order $m$, and has $q^{\prime \prime} \geqq q^{\prime}-q$ different poles $z_{1}, z_{2}, \cdots, z_{q^{\prime \prime}}$ of order $m$ on $|z|=r$. Hence, as was shown in $\$ 4$ (see (16)) $\alpha_{n}$ has the form

$$
\alpha_{n}=n^{m-1} \sum_{k=1}^{q^{\prime \prime}} \frac{\theta_{k}}{z_{k}^{n}}+O\left(\frac{n^{m-2}}{r^{n}}\right)
$$

as $n \rightarrow \infty$, and $\theta_{k} \neq 0\left(k=1,2, \cdots, q^{\prime \prime}\right)$.

It is easily shown ${ }^{4}$ that there is a positive number $M$ such that, for $n=0,1, \cdots$, at least one of the $q^{\prime \prime}$ numbers $\left|r^{n+\nu} \sum_{k=1}^{q^{\prime \prime}} \theta_{k} / z_{k}^{n+\nu}\right|$, $\nu=0,1, \cdots, q^{\prime \prime}-1$ is greater than $M$. This fact, together with (29), implies that, to any given $n_{0}$, there are numbers $n>n_{0}$ such that $\left|\alpha_{n}\right|>(1 / 2) M n^{m-1} r^{-n}$, whereas, because of (28), $n_{0}$ may be found such that, for all $n>n_{0},\left|\alpha_{n}\right|<(1 / 2) M n^{m-1} r^{-n}$. Thus the assumption $q^{\prime}>q$ is proved to be wrong.

Since we have proved that the number of poles of $f(z)$ of order $m$ on $|z|=r$ is $q$, the other statements of the theorem follow immediately from Theorem 2 .

COROLLARY. If $f(z)=\sum_{n} a_{n} z^{n}$ has no singularities but poles on the circle of convergence $|z|=r$ of $\sum_{n} a_{n} z^{n}$, and if $z_{1}=\lim _{n \rightarrow \infty} a_{n} / a_{n+1}$ exists, then $f(z)$ is of order $m=1+\lim _{n \rightarrow \infty}\left(\log \left|a_{n}\right|+n \log r\right) / \log n$ on $|z|=r$, $z_{1}$ being the only pole of order $m$ on $|z|=r .5$

This is obviously Theorem 4 for $q=1$.

The following theorem is the logical product of Theorems 3 and 4.

THEOREM 5. Suppose that, for a fixed $p$, there are $p$ numbers $\gamma_{1}, \gamma_{2}, \cdots, \gamma_{p}$ and a number $\tau>1$ such that, for $i=1,2, \cdots, p$, $\gamma_{i}+d_{n i}^{(p)}[f] / d_{n}^{(p)}[f]=O\left(\tau^{-n}\right)$, as $n \rightarrow \infty$; suppose that there is no $p^{\prime}<p$ such that the limits $\lim _{n \rightarrow \infty} d_{n i}^{\left(p^{\prime}\right)}[f] / d_{n}^{\left(p^{\prime}\right)}[f]\left(i=1,2, \cdots, p^{\prime}\right)$ exist. Then $f(z)$ is regular in the circle $|z| \leqq r=\left|\gamma_{p}\right|^{1 / p}$ but for $p$ poles of the first order on $|z|=r$, which are the zeros of the polynomial $\pi(z)$ $=z^{p}+\sum_{i=1}^{p} \gamma_{i} z^{p-i}$.

4 See A. Ostrowski, Über Singularitäten gewisser mit Lücken behafteten Potenzreihen, Jber. Deutschen Math. Verein. vol. 35 (1926) p. 269.

$\checkmark$ Cf. footnote 3. 
Proof. As in the proof of Theorem 3 it is seen that $f(z)$ has no other singularities than, at most, $p$ poles (counted according to their multiplicities) on the circle of convergence $|z|=r$ of $f(z)=\sum_{n} a_{n} z^{n}$. If, on $|z|=r, f(z)$ had less than $p$ poles, or if some of the poles were of higher than the first order, then $f(z)$ would have $q<p$ poles of highest order on $|z|=r$. This would imply, by Theorem 2 , the existence of the limits $\lim _{n \rightarrow \infty} d_{n i}^{(q)} / d_{n}^{(q)}(i=1,2, \cdots, q)$, in contradiction with the hypothesis of the theorem. $f(z)$ has, therefore, exactly $p$ poles of the first order on $|z|=r$. The other statements of the theorem follow immediately.

7. Application to the evaluation of roots. The zeros of smallest absolute value of the function $F(z)=\sum_{n} A_{n} z^{n}$ are the poles of the function $f(z)=\sum_{n} a_{n} z^{n}=1 / F(z)$ on its circle of convergence. Hence the theory just developed contains a method of evaluating zeros of smallest absolute value of functions given by Taylor series. The procedure to follow is that outlined at the beginning of $\$ 6$. It requires the evaluation of the determinants $d_{n i}^{(m)}[f](n=0,1, \cdots)$ for several values of $m$. These determinants are defined, in (8), in terms of the coefficients $a_{n}$ of $f(z)$. They also can be expressed ${ }^{6}$ easily in terms of the coefficients $A_{n}$ of the given function $F(z)$, with the determinant $d_{n i}^{(m)}[f]$ of order $m$ in the elements $a_{n}, a_{n+1}, \cdots, a_{n+2 m-1}$ replaced by a determinant of order $m+n+1$ in the elements $A_{0}, A_{1}, \cdots, A_{n+2 m-1}$.

Since the order of these latter determinants increases with $n$, they are not suited for practical purposes. If numerical evaluation of the zeros of $F(z)$ is required, it is advisable to compute first the coefficients $a_{n}$ of the reciprocal function $f(z)$, and then to work with the determinants $d_{n i}^{(m)}[f]$ as given in (8). If $f(z)=\sum_{n} a_{n} z^{n}$ is the negative reciprocal of $F(z)=\sum_{n} A_{n} z^{n}$, and the coefficient $A_{0}$ is made to be -1 , then the coefficients $a_{n}$ are given by the simple recursion formulae:

$$
\begin{aligned}
& a_{0}=1, \\
& a_{1}=A_{1}, \\
& a_{2}=A_{2}+a_{1} A_{1}, \\
& a_{3}=A_{3}+a_{1} A_{2}+a_{2} A_{1},
\end{aligned}
$$

To illustrate the numerical efficiency of the method, the two conjugate complex roots $z_{1}, z_{2}$ of smallest absolute value of $F(z)=z-e^{z}=0$ were computed. The results are exhibited in the following table. The

${ }^{6}$ See J. Hadamard, loc. cit., p. 136. 
second column of the table contains the Taylor coefficients $a_{n}$ of $f(z)=1 /\left(e^{z}-z\right)$ multiplied by $n$ ! in order to avoid fractions; the third column contains the quotients $d_{n 1}^{(2)}[f] / d_{n}^{(2)}[f]$, which represent successive approximations to $-\gamma_{1}=z_{1}+z_{2}$; the fourth column contains the quotients $d_{n-1}^{(2)}[f] / d_{n}^{(2)}[f]$, which represent successive approximations to $\gamma_{2}=z_{1} z_{2}$. The final values in the table give

$$
z_{1}+z_{2}=0.6362630104, \quad z_{1} z_{2}=1.889406976
$$

with a relative error of $1 \cdot 10^{-10}$. As the table shows, the fifth approximation, which is obtained with very little effort, already gives the values of $z_{1}+z_{2}$ and $z_{1} z_{2}$ with a relative error of $1 \cdot 10^{-6}$.

\begin{tabular}{|c|c|c|c|c|c|c|}
\hline$n$ & \multicolumn{4}{|c|}{$a_{n}$} & $d_{n 1}^{(2)}[f] / d_{n}^{(2)}[f]$ & $d_{n-1}^{(2)}[f] / d_{n}^{(2)}[f]$ \\
\hline 1 & & & & 0 & 0.67 & 2 \\
\hline 2 & & & & -1 & 0.632 & 1.89 \\
\hline 3 & & & & -1 & 0.6368 & 1.891 \\
\hline 4 & & & & 5 & 0.63616 & 1.8897 \\
\hline 5 & & & & 19 & 0.6362624 & 1.88942 \\
\hline 6 & & & & -41 & 0.6362628 & 1.88941 \\
\hline 7 & & & & -519 & 0.63626291 & 1.8894073 \\
\hline 8 & & & & -183 & 0.63626306 & 1.8894072 \\
\hline 9 & & & 19 & 223 & 0.63626299 & 1.88940700 \\
\hline 10 & & & 73 & 451 & 0.636263011 & 1.889406978 \\
\hline 11 & & & -847 & 067 & 0.6362630099 & 1.889406977 \\
\hline 12 & & -8 & 554 & 547 & 0.6362630105 & 1.889406976 \\
\hline 13 & & 32 & 488 & 611 & 0.6362630104 & 1.889406976 \\
\hline 14 & & 977 & 198 & 559 & 0.6362630104 & 1.889406976 \\
\hline 15 & 1 & 325 & 135 & 969 & & \\
\hline 16 & -116 & 987 & 762 & 287 & & \\
\hline
\end{tabular}

Purdue University 